\title{
Cuestiones para investigación: ¿Qué pasa con los servicios sociales en España?
}

\author{
Questions to be investigated: What has happened \\ with the social services in Spain?*
}

\section{Belén Morata García de la Puerta**}

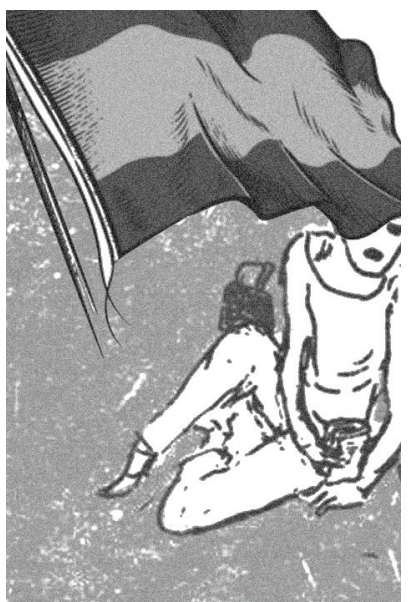

Resumen: El propósito de este artículo es explicar qué está pasando con el actual sistema público de servicios sociales en España. El contexto de bienestar mediterráneo español en el que se insertan los servicios sociales se desarrolló más tarde que en otros países del entorno inmediato. Antes de alcanzar la suficiente madurez y desarrollo se identifican una serie de obstáculos y déficits legales y políticos, que ponen en duda la efectividad del principio universalista de los servicios sociales y su posición real en el sistema de bienestar

Palabras-clave: Servicios sociales. España. Derechos sociales. Bienestar social. Principio de universalidad. Pobreza y exclusión.

\begin{abstract}
The purpose of this article is to explain what has been happening in the current public social service system in Spain. The Spanish Mediterranean welfare context in which social services are inserted, was developed later than in other countries in the immediate environment. Before reaching sufficient maturity and development we can identify several legal and political obstacles and deficiencies that cast doubts on the effectiveness of the universal principles of social services and their actual position in the welfare system
\end{abstract}

Keywords: Social services. Spain. Social rights. Social welfare. Principle of universality. Poverty and exclusion.

* Texto corrigido por Sandra Valeuzuela.

** Profesora de Servicios Sociales de la Facultad de Trabajo Social, Universidad de Granada — Granada, España.E-mail: bmorata@ugr.es. 


\section{Introducción}

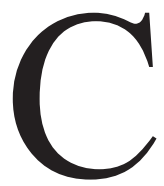

uando España regresa formalmente a la democracia, en 1978, parecía que el nuevo valor ideológico sobre el que se iba a asentar la cobertura de necesidades sociales daría un giro completo al sistema asistencial vigente en aquellos momentos. Es cierto que los servicios sociales que se crean a partir de la década de los 80 del siglo pasado se hicieron sobre las viejas estructuras franquistas y, en realidad, mucho más antiguas en el caso de la atención a personas mayores, personas con discapacidad y menores, pues se remontan en el tiempo a varios siglos atrás. A pesar de ello, los principios inspiradores de la Constitución española parecían indicar que los servicios sociales que surgirían superarían el contexto autoritario y paternalista del franquismo, hacia el reconocimiento de verdaderos derechos sociales, como derechos de ciudadanía.

Como veremos más adelante, los servicios sociales que surgen de la democracia han experimentado una transformación espectacular desde el franquismo y la transición democrática al momento actual. ${ }^{1}$ Esto nadie hoy lo pone en duda. Sin embargo, este cambio no ha conseguido desprender totalmente algunos de los rasgos definidores del modelo que lo precedió. La creación de un sector autónomo de la política social, como uno de los pilares del Estado de bienestar español, junto a la sanidad, la garantía de rentas o la educación es relativamente reciente, se ha fraguado en los últimos treinta años de historia.

El propósito de este artículo es describir cómo surgieron los servicios sociales democráticos en España y cuáles son los déficits y retos que tienen planteados en la actualidad. Para ello, se explicará brevemente el contexto de bienestar en el que se inserta este sector de la política social, cómo influye el sistema político español en el desarrollo de los servicios sociales y cuál ha sido el papel que los y las trabajadoras sociales han desarrollado dentro del mismo.

1. Siguiendo a Carme Molinero (2003), el régimen franquista trajo consigo el restablecimiento de los fundamentos básicos de la sociedad burguesa (propiedad privada, orden clasista y jerarquía social). La represión se utilizó como instrumento de control social y al mismo tiempo, resaltó la función de control de la política de asistencia social. A pesar de que el discurso sobre "la preocupación social" fue un elemento de referencia política y uno de los instrumentos preferidos de la propaganda del Estado, lo cierto es que las partidas destinadas a gasto social tuvieron muy poca relevancia hasta bien entrada la década de los 60 y aún así, muy escasas en comparación con los países europeos de nuestro entorno. La asistencia social no era concebida como un derecho de los individuos, sino como producto de la "conciencia social" de la revolución nacional. Esto significaba que la asistencia a los necesitados se realizaba con miras a la reconstrucción nacional, con una dosis fortísima de adoctrinamiento, coacción y con frecuencia menosprecio a quienes no comulgaban con la ideología única del régimen. 
Aunque algunos de los déficits y dificultades actuales son compartidos por otros países de nuestro entorno inmediato - y tienen que ver fundamentalmente con las transformaciones profundas de las sociedades occidentales, a lo largo de los últimos años, que han provocado la aparición de nuevos riesgos sociales - sin embargo, algunas características son específicas de la realidad española. A ellas me voy a referir de manera especial.

\section{El modelo de bienestar español}

Los problemas y déficits que aquí se plantean no están, obviamente, desmarcados del modelo de bienestar en el que se insertan los servicios sociales. Desde la ampliación de la clasificación que hiciera Esping-Andersen sobre los Regímenes de Bienestar y la incorporación de los países de la Europa del Sur (España, Grecia, Italia y Portugal), la literatura ha identificado los elementos sustanciales del régimen de asistencia social latinomediterráneo. Siguiendo a Adelantado, Moreno y Recio (2007), ${ }^{2}$ los rasgos son los siguientes:

1. Es un sistema dualista de mantenimiento de rentas, donde las prestaciones más elevadas se asignan a los grupos sociales con fuerte relación con el mercado de trabajo formal. Las prestaciones discrecionales o escasas van a parar al resto de la población (Ferrera, 1995).

2. Inexistencia de una red pública de garantía de ingresos: ausencia de programas nacionales de asistencia que provean de prestaciones a todas las personas que carecen de recursos suficientes: no hay una protección legal ni se han establecido criterios para delimitar un nivel mínimo de subsistencia (Gough, 1996).

3. La oferta pública de recursos residenciales (centros de día, ayuda a domicilio, residencia) se sitúa muy por debajo de la media europea. La familia, y especialmente las mujeres, realizan labores de cuidado de las personas dependientes. Las prestaciones asistenciales para mayores o personas dependientes, familias y personas en situación de desempleo son muy bajas en el ámbito de la Seguridad Social.

2. Los autores que aparecen a continuación están citados en el Trabajo de Adelantado, Moreno y Recio: "Informe sobre la política autonómica de servicios sociales en España y Cataluña", del IGOP de la Universidad Autónoma de Barcelona. 
4. El acceso a las prestaciones se hace previa comprobación de inexistencia de recursos, la financiación al sistema es limitada y se deja un amplio margen de intervención al sector privado lucrativo y a la inciativa social sin ánimo de lucro (Sarasa y Obrador, 1999).

5. El acceso y la garantía a las prestaciones se condicionan a la disponibilidad de recursos y no existen verdaderos derechos subjetivos que amparen a los ciudadanos, dejando en papel mojado el "derecho a acceder al sistema público de servicios sociales". Esta última característica afecta especialmente al tema que nos ocupa y que se desarrollará en las próximas páginas.

Estas características son propias de los países del sur de Europa y por esta razón no se adaptan exactamente a los modelos clásicos de bienestar. En realidad, el modelo mediterráneo se considera un modelo intermedio respecto a otros regímenes de protección social de las democracias avanzadas, al haber alcanzado un nivel intermedio de desmercantilización (de-commodification) y de acceso universal por comprobación de medios (means testing) a prestaciones y servicios sociales (Moreno, 2001).

Hablamos, en consecuencia, de un cuarto modelo, propio de Europa meridional que comparte una serie de analogías respecto de la historia, del sistema de valores así como de sus peculiaridades institucionales: con distinto grado y duración, estos países sufrieron dictaduras y gobiernos autoritarios durante el siglo XX. Además, la Iglesia Católica ha sido uno de los principales agentes proveedores de bienestar. Por otra parte, la pertenencia a la Unión Europea (y también por efecto de la Globalización) ha fomentado una constante convergencia con los países del centro de Europa y del área septentrional.

$\mathrm{Al}$ igual que en Grecia, Italia y Portugal, el papel que ha desarrollado la familia en el régimen de bienestar español es un factor estructural que interacciona con el Estado y los poderes públicos y también con las instituciones de la sociedad civil. La familia actúa como un colchón que amortigua los efectos del infortunio en los casos de desempleo, enfermedad, invalidez de sus miembros. Incluso en España la garantía de algunos derechos sociales aparece supeditada a las obligaciones legales de la familia (Morata et al., 2010). ${ }^{3}$ Esta situación permite a los jóvenes prolongar el tiempo de dependencia económica de sus familias mucho más que en el resto de los países del entorno, retrasando la edad de la emancipación. Quizá esto podría explicar por qué las políticas de juventud españolas han ido

3. El llamado "Derecho de alimentos" supedita algunas ayudas y prestaciones públicas a la inexistencia de parientes cercanos que, según la ley, deban y puedan hacer frente a sus responsabilidades familiares. 
paulatinamente aumentando la edad de sus beneficiarios, a quienes se sigue considerando jóvenes a pesar de haber cumplido los 30 años.

Como en el resto de Europa el régimen de bienestar se ha asentado en una serie de pilares, que son la educación, la sanidad, la garantía de rentas y los servicios sociales. Desde luego, podría afirmarse que uno de los factores que contribuyeron positivamente al diseño del modelo de bienestar actual fue el que gobernara ininterrumpidamente, durante más de una década, el partido socialista (PSOE - Partido Socialista Obrero Español). A lo largo de sus mandatos se fraguaron los sectores de la política social. La experiencia española nos muestra que cuando desde el poder público se ha impulsado un sector, apostando por políticas de carácter universalista (el caso de la sanidad y educación), el resultado ha sido muy notable. Por el contrario, si el sector se ha basado en la focalización de la atención ante los problemas, así como el carácter subsidiario del impulso estatal (el caso de los servicios sociales), los resultados han sido muy limitados. A continuación, se mostrarán algunos de los factores que han influido y configurado su situación actual.

\section{El modelo de gestión multinivel de servicios sociales}

El modelo de Estado que surge a partir de 1978 - el Estado de las Autonomías, también llamado Estado Autonómico español se ha calificado como un modelo híbrido o a medio camino entre un Estado federal y un Estado unitario. Solozábal (1992) explicó en su momento cuáles fueron las razones ideológicas que provocaron este diseño, que se remontaban a varios siglos atrás, y cómo se articuló desde el punto de vista jurídico y político. De manera sencilla cabe decir que fue un modelo de consenso que intentó conjugar las aspiraciones autonomistas (incluso, independentistas) de algunos sectores de la sociedad con la unidad del Estado, a la manera tradicional española. El reconocimiento de la pluralidad de pueblos que integran el Estado fue plasmado en la idea de "autonomías y nacionalidades", como organizaciones territoriales dotadas de poder político. De esta manera, las regiones y algunas provincias accedieron al autogobierno, y se homogeneizó el término de Comunidades Autónomas para denominarlas a todas ellas.

La Constitución de 1978 repartió las competencias sobre las materias entre el Estado y las recién creadas Comunidades Autónomas, ${ }^{4}$ sin incluir en este re-

4. La Constitución española reconoce al Estado la competencia de garantizar la asistencia y prestaciones sociales suficientes ante situaciones de necesidad, especialmente en los casos de desempleo (con el régimen público de Seguridad Social, principalmente). También reconoce a las Comunidades Autónomas su compe- 
parto a las ya existentes corporaciones locales (Ayuntamientos y Diputaciones, principalmente) aunque después su papel ha sido fundamental en la gestión del bienestar en todos los municipios de España. La Constitución pospone este tema a la legislación que posteriormente apruebe el Estado y las Comunidades autónomas en sus territorios para concretar qué materias serán de responsabilidad de las entidades locales y en qué medida ejecutarán sus competencias. Esto significa que, aunque la Constitución atribuya a las Comunidades Autónomas la responsabilidad exclusiva de los servicios sociales en su territorio, el Estado podría haber aprobado una Ley Básica (o una Ley Marco) de Servicios Sociales que hubiera servido para homogeneizar el sistema público que cada Comunidad Autónoma habría de crear. Pero es más; esta Ley podría aclarar y definir algunas de las cuestiones que desde hace treinta años han limitado y perjudicado el desarrollo de este pilar del Estado de Bienestar. Sin embargo esto no se hizo y es, quizá, una característica típica española que se resume brevemente: la permanente indefinición.

Tenemos en España un modelo multinivel de gestión del bienestar en el que participan el Estado, las Comunidades Autónomas pero - aunque en la práctica quienes gestionan los servicios sociales son los municipios - no se ha hecho la verdadera descentralización en ellos. No son los titulares de las competencias y dependen del Estado y de la Comunidad Autónoma correspondiente, en gran medida, para sufragar el coste de estos servicios públicos, pues su capacidad recaudatoria está muy limitada por la legislación. Quizá la coyuntura política del momento arroje una luz al tema, pues la carrera de las Comunidades Autónomas por aprobar leyes de servicios sociales a partir de 1982 (desplegándose una primera fase de legislación autonómica completa) fue suficiente como para que el Estado desistiera de la posibilidad de aprobar una ley general que permitiera homogeneizar la legislación autonómica y, especialmente, que dejara claro algunos temas importantes, desde el punto de vista de la dimensión territorial y sobre el propio contenido de los servicios sociales: qué clase de derecho se reconoce a la ciudadanía. Si es un derecho genérico (derecho a acceder al sistema público) o es un derecho a las prestaciones concretas del sistema. Cuáles serían las condiciones de acceso, cuál sería el catálogo de prestaciones sociales mínimas y obligatorias en todos los municipios que por ley deben prestar servicios sociales etc.

tencia en materia de asistencia social. Al mismo tiempo, hay una mención expresa a la responsabilidad estatal de garantizar la igualdad de todos los españoles y el control de algunas materias, como la inmigración, asilados y refugiados, ámbito penitenciario (excepto en Cataluña) y sobre todo, la regulación básica y el régimen económico de la Seguridad Social y del desempleo. 
Probablemente el momento político no era el más adecuado pues, como bien indican Adelantado y Gomá (2000), la necesidad de legitimación que tienen los recién creados gobiernos autonómicos, les empuja a acaparar cuantos servicios les doten de visibilidad ante sus ciudadanos. Al mismo tiempo, existe una importante conflictividad judicial entre el Estado y las Comunidades Autónomas, en una disputa por el alcance y contenido de las competencias. A ello habría que añadir la excesiva fragmentación de los municipios españoles que deja a buena parte de ellos con un tamaño insuficiente para gestionar de manera eficaz los servicios.

Al contrario que la sanidad y la educación en España, en donde sí existe legislación básica general del Estado, los servicios sociales quedaron en el ámbito exclusivo de las CCAA. En el año 1988, se aprueba un convenio marco entre Estado y Comunidades Autónomas, el llamado Plan Concertado para el desarrollo de las prestaciones básicas de servicios sociales en las corporaciones locales, que ha sido el único intento del Estado para impulsar, con carácter global, la creación de servicios sociales comunitarios, en los municipios de más de 20 mil habitantes. La pretensión de este impulso estatal se concreta en el establecimiento de unas prestaciones básicas y unos equipamientos básicos, comunes a todo el territorio español. Al mismo tiempo se establecen una serie de principios, que serán asumidos al firmar el convenio, a propósito de la financiación, de la cooperación y colaboración que debe presidir el desarrollo del convenio por parte de las administraciones públicas implicadas.

Toda esta problemática, de carácter legal y técnico, excede a las posibilidades de este artículo, pero he querido detenerme brevemente en ello porque ha sido uno de los principales handicaps, como reconocen los propios directores y responsables técnicos de los centros de servicios sociales comunitarios. Existe una importante producción legislativa autonómica pero muy escasa o nula regulación estatal, quedando a merced de las Comunidades Autónomas y con un verdadero problema de cooperación y colaboración (y a veces de entendimiento, por discrepancias políticas entre los partidos diferentes que gobiernan la Autonomía y el Ayuntamiento o la Diputación). La existencia de un modelo de relaciones intergubernamentales entre los niveles implicados podría haber mejorado la práctica diaria de la gestión de políticas públicas en las que simultáneamente deben intervenir varias Administraciones. Finalmente, la autonomía política ha dado lugar a una heterogeneidad de los sistemas de servicios sociales en las Comunidades Autónomas. En estos momentos existen claras diferencias en los distintos territorios de España, condicionadas, entre otras, por la ideología de los partidos gobernantes, las prioridades de las agendas políticas autonómicas y, cómo no, las 
diferencias entre Comunidades Autónomas ricas y pobres. Volveremos sobre este tema en páginas posteriores.

\section{Principales características del sistema público de servicios sociales}

Desde la década de los 80 se ha hablado de los servicios sociales como el sexto sistema, junto a la educación, salud, vivienda, garantía de rentas y seguridad social, si bien ocupó inicialmente un lugar residual en la literatura especializada (Kahn y Kanerman, 1987; Bueno Abad, 1990; Adelantado, 2007). El sistema de servicios sociales debiera ser universal e igual para todos, cubrir aquellas necesidades no atendidas por el resto de sistemas. Cada uno de ellos se ocuparía de sus objetivos y el sexto sistema sólo se encargaría de la información y la formación, la promoción de la convivencia, la solidaridad y la integración social. De esta manera, cada uno asumiría sus responsabilidades, como partes de una estructura de bienestar que atendiera cualquier tipo de necesidad (Sanz Citora, 2001).

Así, los servicios sociales se institucionalizan - como sistema diferenciado - de carácter público, estructurado en dos niveles de atención (atención primaria y nivel especializado), siendo los Ayuntamientos democráticos quienes debían asumir la responsabilidad de su desarrollo, con la ayuda de los otros niveles de gobierno. El sistema se compone de diferentes prestaciones y servicios (prestaciones técnicas, prestaciones económicas, centros e instituciones, programas, proyectos y actividades). Se trata de un conjunto de elementos y prestaciones que están interrelacionados y que, de manera coordinada, van dirigidas a un mismo fin (Pelegrí, 2007).

El concepto de "básico" recae sólo sobre algunas necesidades y para ellas se diseñaron una gama de prestaciones (información y orientación, convivencia personal, integración social y cooperación y solidaridad social) y equipamientos necesarios (centros de servicios sociales, albergues y centros de acogida) que se gestionaban desde los ayuntamientos. A pesar de ello, han subsistido las diferencias regionales, en la calidad, cantidad y formas de gestión. Ya se ha aprobado la segunda generación de leyes autonómicas de servicios sociales, sin que con ello se haya avanzado en la concepción de los servicios sociales. Las opciones políticas de servicios sociales y las agendas institucionales son ciertamente diferentes. Hubo que hacer un esfuerzo financiero y organizativo y esto ha dependido de las prioridades políticas de los gobiernos de las Comunidades Autónomas 
Tabla 1

Niveles de atención de los servicios sociales ${ }^{5}$

ATENCIÓN PRIMARIA O COMUNITARIA: es el primer nivel de atención y más cercano a la ciudadanía por su implantación territorial. Atiende todas las situaciones sociales de la población de una zona. Las prestaciones de este nivel de atención son:

1. Información, orientación, asesoramiento, valoración, canalización y derivación a otros dispositivos sociales.

2. Apoyo a la unidad de convivencia y ayuda a domicilio.

3. Alojamiento alternativo, de carácter temporal o permanente.

4. Prevención e inserción.

5. Fomento de la solidaridad y cooperación social, para favorecer los procesos de participación y cooperación de las personas.

ATENCIÓN ESPECIALIZADA: la atención se concreta en diferentes colectivos o sectores de población en función de sus características o problemática determinada. Se estructura en servicios interprofesionales, con una implantación en el territorio menos sistemática que el nivel anterior. Disponen de equipamientos y equipos de expertos para atender a los colectivos o sectores.

Fuente: Elaboración de la autora.

Durante la transición democrática, se discutieron ampliamente qué características y qué naturaleza debiera regir el sistema. Hubo un debate teórico e ideológico de rechazo frontal a la beneficencia y al asistencialismo del momento. El debate giraba en torno a la configuración de este modelo tomando como punto de referencia el de los países desarrollados de nuestro entorno. Predominaron las tesis que incluían la universalidad de los servicios como un principio inspirador del modelo, que se basaría en una atención preventiva y el desarrollo comunitario. Una nueva ideología democrática impregnaría a los servicios sociales, al querer huir del asistencialismo discrecional público para centrarse en el cumplimiento de los derechos de ciudadanía, por parte de las administraciones públicas.

Pero este modelo ha quedado más bien en la teoría que en la práctica. Las leyes de servicios sociales que fueron aprobando las Comunidades Autónomas recogieron en sus preámbulos los principios de universalidad y gratuidad del sistema, junto a otros. Éstos no llegaron a desarrollarse en el texto y, en consecuencia, su efectividad quedó limitada a una mera expectativa de derecho, más a una posibilidad que a una realidad. Se impusieron, sin embargo, una serie de factores que

5. A partir de 1995 hay un acuerdo de flexibilización de servicios, admitiendo equipamientos complementarios, tales como: comedores sociales, pisos tutelados, minirresidencias, oficinas o servicios de información y centros de estancia diurna. 
interfirieron en el desarrollo de este modelo; debe resaltarse, en primer lugar, la falta de voluntad política por considerar al sistema público de servicios sociales en pie de igualdad a los otros pilares del Estado de Bienestar. Una parte importante de la clase política española participa de una visión malthusiana de la pobreza y, probablemente, también un sector apreciable de la opinión pública española comparte este sentir. Como consecuencia de ello, ha persistido una concepción de los servicios sociales anclada al pasado, como una práctica tradicional de beneficencia, que se da o se retira en función de distintos criterios coyunturales, como puede ser la disponibilidad presupuestaria o las propias prioridades de la agenda política del gobierno, cuando no la crisis económica.

Y prueba de ello es que, a pesar del tiempo transcurrido, seguimos parados en un momento de desarrollo inicial del sistema público de servicios sociales. De hecho, parece haber en un equilibrio inestable y dinámico entre el sistema prestacionista y el impulso transformador de la sociedad. Así, esta ambivalencia se manifiesta en que, por una parte, es un modelo basado en reglas de funcionamiento, en la gestión de prestaciones económicas que pueden generar dependencias institucionales, en las normas del sistema y la experiencia acumulada, y en la existencia de servicios como centro del sistema y puerta de entrada al conjunto de recursos públicos. Pero, al mismo tiempo, es un modelo centrado en las familias y libre de condicionamientos burocráticos de funcionamiento, flexible y experimental, centrado en procesos educativos y promocionales y abierto a la colaboración con el conjunto de recursos y profesionales de los barrios (Sanz Citora, 2001).

Se ha hecho un esfuerzo importante durante estos años, de manera que hoy encontramos en los servicios sociales equipamientos, tales como: centros de acogida, residencias, pisos tutelados, servicio de atención a domicilio y centros de atención a drogodependientes. Sin embargo, la oferta pública de servicios sociales española es muy reducida, situándose por debajo de la media europea, por lo todavía existe una importante participación de la familia, en quien recae la función de cuidados y en las redes sociales de proximidad. Hay un sector creciente de atención de carácter privado y voluntario (organizaciones no lucrativas y religiosas) aunque es muy deficiente el sistema de coordinación y concertación entre el sector público y el sector privado (Sarasa, 1995). ${ }^{6}$ No existe en España un modelo de colaboración público privada en la atención a necesidades sociales. Nuevamente, las Comunidades Autónomas han creado un sistema heterogéneo y hay partes del territorio español en donde la oferta privada de servicios predomina frente al sector público en la prestación y gestión de muchos de los servicios sociales, no siempre bien

6. En Subirats et al., 2007. 
regulada ni inspeccionada. Algunos modelos, como el andaluz, mantiene la titularidad pública, pero incluye dentro de la oferta de servicios y prestaciones del sistema público de servicios sociales, tanto a la red pública como a la concertada y también la privada.

En algunas Comunidades Autónomas, incluso, se observa una paulatina externalización de servicios, pues el protagonismo que ha adquirido la iniciativa privada desplaza a un papel residual a la actuación pública, sólo para determinados colectivos de población muy concretos. La función de promoción del bienestar, de cohesión social y solidaridad deja de tener sentido y parece predominar una visión de la política social como instrumento de control, para garantizar la paz social.

En el año 2006, el gobierno de España aprueba una ley de promoción de la autonomía personal y atención a las personas en situación de dependencia. Pese a que el colectivo de beneficiarios de esta ley está acotado (personas que necesitan de la ayuda y atención por razones de edad o enfermedad) se produce un punto de inflexión en el ámbito de los servicios sociales, por distintos motivos: en primer lugar, porque esta ley recogerá para los beneficiarios verdaderos derechos subjetivos a la atención de su situación y a la promoción de la autonomía personal. La ley desarrolla una cartera de servicios y prestaciones concretas a las cuales las personas - a las que la administración pública reconoce su dependencia - con independencia de su renta económica o cualquier otra situación tienen derecho. En consecuencia, se reconoce con carácter universal el derecho a la promoción y a la atención.

En segundo lugar, se trata de una ley estatal que se aplicará en todo el territorio de la nación. Se reconoce un mínimo obligatorio en cualquier parte del Estado, que luego puede ser mejorado y ampliado por las Comunidades Autónomas. Además de ello, se crea, dentro del sistema público de servicios sociales (y no dentro de la Seguridad Social, como en otros países del entorno) la acción protectora del sistema. ${ }^{7}$ Por último, junto al desarrollo de derechos sociales individuales se ha apostado por el sistema al dotarlo de los recursos necesarios para su implementación.

Este sistema de atención a la dependencia puede ser un impulso importante para el sistema de servicios sociales autonómico. A partir de su aprobación, de manera tímida, algunas Comunidades Autónomas han reformado sus leyes de Servicios Sociales, incorporando una cartera de servicios para la ciudadanía y regulando rentas mínimas de inserción a las personas residentes que se encuentren en

7. Sistema para la autonomía y atención a la dependencia (SAAD), que se constituye por 3 niveles de atención, con participación también del sector privado - lucrativo y no lucrativo - , cuyos servicios se prestarán a través de la red pública de servicios sociales, con independencia de la titularidad de los mismos. 
situación de necesidad y tengan rentas económicas por debajo del baremo establecido. Son muy pocas las Comunidades Autónomas que están regulando este tipo de rentas, que se gestionan desde los servicios sociales, como última red de seguridad para familias expulsadas del sistema de protección social y en situación de pobreza y/o exclusión social. Sin embargo, es de esperar que sirva como revulsivo para superar esa posición secundaria y subsidiaria del conjunto de sistemas de protección social del Estado.

\section{El papel de los y las trabajadoras sociales}

En este contexto, la profesión de trabajador/a social, como una profesión de ayuda, intenta tomar protagonismo y conciencia en el desarrollo de sus funciones en los servicios sociales.

Desde los años 80, la profesión experimentó un desarrollo importante ligado al impulso y despegue de los servicios sociales. La literatura especializada, inmersa también en este proceso de institucionalización, contribuye al mismo aunque sin entrar, con carácter general, en el fondo del problema de la efectividad del principio universalista de los servicios sociales y su posición real en el sistema de bienestar. Aspectos tales como la estructura organizativa, la legislación, la delimitación conceptual, el desarrollo de ámbitos competenciales propios, sectores especializados de atención, el desarrollo de las prestaciones básicas de servicios sociales, entre otros, copan las aportaciones teóricas, lo que era coherente con un momento en el que había que justificar la creación del sistema, un espacio competencial propio y reforzar nuevo valor ideológico, fundamento de la cobertura de necesidades sociales (Morata et al., 2009).

Como se ha adelantado antes, a pesar del impulso, político, legislativo y doctrinal, muy pronto empiezan a surgir las voces que hablan de la contradicción manifiesta entre el "voluntarismo idealista" de la legislación de servicios sociales y la práctica cotidiana. Las explicaciones que se han dado a este fenómenoapuntan a la concurrencia de distintos factores, en muchas ocasiones, de manera simultánea:

- la potencialidad del sistema público de servicios sociales se desactiva durante su puesta en marcha, en plena transición política, por la dejadez de funciones de las Corporaciones Locales (Casado, 2001);

- el desarrollo de los servicios sociales está directamente ligado al modelo de intervención social, basado principalmente en la prestación de servicios 
y que hace primar la atención personal al usuario en los despachos, y las prestaciones técnicas y económicas. Especialmente, la comprobación de medios, como requisito previo para recibir las prestaciones, distrae al trabajador/a social en labores rutinarias y burocráticas — que además de no ser propias de su condición - lo aleja de los procesos educativos y de cambio de actitudes, que sí son más acordes a la función que el Trabajo Social debe llevar consigo (Báñez, 1995 );

- el tipo de organización de los servicios sociales es más fragmentada que descentralizada y con grandes déficits de coordinación entre niveles intra e intersectoriales (Adelantado et al., 2007);

- es difícil, desde los entes locales, desarrollar acciones de promoción o rehabilitadoras y mucho menos de tipo terapéutico, por cuanto que los usuarios o "clientes" de los servicios sociales sólo esperan prestaciones materiales, con carácter puntual, como pueden ser las prestaciones económicas, los recursos residenciales y acceso a la vivienda, entre otros (CarpenteryTreacher, 1993);

- la visión actual de los servicios sociales es la de servicios para colectivos vulnerables, para determinadas categorías especiales de personas. A diferencias de los servicios educativos o sanitarios (pensados para cualquier persona que pueda necesitarlos en diferentes etapas o contingencias de la vida) éstos se adscriben a un segmento de población estático y definido en función de una única característica humana (Fantova, 2008).

El papel que desarrollan los y las trabajadoras sociales está muy condicionado por la estructura y funcionamiento de los servicios sociales, y que pueden resumirse de la siguiente manera (Defensor Andaluz, 2005 $)$ : hay un excesivo protagonismo de las prestaciones económicas frente a las intervenciones psicosociales o sociosanitarias. La gestión de prestaciones económicas se ha convertido en una de las tareas básicas de los profesionales de los servicios, que se come todo el tiempo y no deja espacio para el trabajo social. Y esto contribuye a la percepción de los profesionales de ser meros gestores de recursos. Los servicios sociales han entrado en un momento de estancamiento, inmersos en un círculo vicioso. El desencuentro entre lo político y lo técnico, con una práctica política malentendida, por encima

8. Báñez, T. (mayo/1995). Situación profesional a la que nos enfrentamos hoy. I Jornadas de Trabajo Social. (Mimeo.)

9. Véase también el "Informe sobre la situación económica y social. Consejo Económico y Social de Aragón", en Sanz, 2001. Igualmente, Morán. "El 4 pilar del Estado de Bienestar está cojo", en Diario de Sevilla, edición digital, 12 sept. 2008. 
de los planteamientos técnicos, ha causado la frustración de muchos profesionales vinculados a los servicios sociales y a la instauración de un sistema de toma de decisiones, basado en la coyuntura frente a la planificación estratégica.

La falta de financiación constante de los programas y actividades, la inestabilidad en el empleo de los propios trabajadores/as sociales que permite a los gobernantes que hagan desaparecer los proyectos que se desarrollarían en zonas marginadas de las ciudades, en momentos de crisis, ha generado un malestar que acaba afectando al funcionamiento mismo de los servicios. Estamos sufriendo un colapso en los servicios sociales pues hay una avalancha de familias que acuden a los servicios sociales en busca de ayuda económica o profesional. En estos últimos años las solicitudes de renta básica para familias que están fuera del sistema de protección social y que carecen de rentas económicas ha aumentado más de un $30 \%$ y hay lista de espera para solicitarlas..$^{10}$

\section{Reflexiones finales: ¿Qué pasa con los servicios sociales en España?}

Para concluir este artículo me gustaría hacer una serie de reflexiones que giran en torno a tres temas y que inciden en la misma idea; el desigual desarrollo del sistema de servicios sociales, como pilar del Estado de bienestar, frente a los otros sistemas mencionados.

En primer lugar, la diferente consideración que se da a los Servicios Sociales frente a los otros pilares del Estado de bienestar claramente ha perjudicado el desarrollo de este sector de la política social. El carácter universalista de éstos, así como la protección legal con la que cuentan, han protegido el sistema frente a los intentos privatizadores de los gobiernos y la tendencia a adelgazar cada vez más el sistema público. La Ley General de Sanidad, en 1986, puso en marcha la reforma y modernización de la sanidad española, que desde ese momento se convertía en universal y con financiación pública a través de los impuestos de todos los españoles, siendo asíun pilar fundamental del Estado de bienestar. Gracias a esta Ley se sustituye la atención benéficoasistencial de la salud por derechos, vinculando la protección sanitaria a la condición de ciudadano. Hoy, la sanidad ocupa el 6,5\% del PIB y aportan más los que más tienen.

10. Declaraciones de la Presidenta del Consejo Nacional de Colegios profesionales de trabajo social al diario El País, 18 ago. 2011. Accesible en: http://www.elpais.com/articulo/sociedad/crisis/derechos/asistenciales/caridad/elpepisoc/20110816elpepisoc_1/Tes. 
De la misma manera, la universalización de la educación se establece a partir de 1985. La enseñanza se hace obligatoria, básica y gratuita sin discriminaciones y queda configurada como otro de los pilares del Estado de Bienestar. El objetivo de garantizar el derecho a la educación y, especialmente el de "educación para la inclusión" están presentes en las agendas gubernamentales. En algunas Comunidades Autónomas, como es el caso de Andalucía, se aplican tanto programas de educación especial como los que tienen en cuenta a las minorías y a otros colectivos desfavorecidos.

La garantía de rentas queda también asegurada gracias a la seguridad social y al sistema de prestaciones por desempleo. En ambos casos, se mantienen líneas de prestaciones no contributivas para quienes no contribuyeron (o lo hicieron de manera insuficiente) a la seguridad social, aunque de menor cuantía y de acceso selectivo, una vez comprobada la situación de necesidad.

Por el contrario, respecto de la protección social, encontramos una dualidad clara en la atención pública, que se manifiesta en una división de la ciudadanía en función de su relación con el mercado laboral y sus rentas que es, realmente, lo que condiciona su acceso a la protección. Los trabajadores y sus familias están bajo el amparo de la seguridad social y los servicios sociales, mientras que quienes no trabajan o perdieron su empleo tienen vetado el acceso a la seguridad social y terminan en los servicios sociales, como última malla protectora frente al infortunio. Los itinerarios y prestaciones económicas establecidas para este segundo grupo no aseguran, con carácter permanente, una renta básica de ciudadanía. En páginas anteriores hablábamos de la visión malthusiana de la pobreza que perdura en buena parte de nuestra clase política y que, desde el punto de vista práctico se traduce en un modelo muy restrictivo de acceso a estas prestaciones, quizá para no desincentivar la obligación de volver al trabajo y a contribuir a las cargas financieras del Estado. Son excepcionales las Comunidades Autónomas que han previsto Programas de Rentas Mínimas, con carácter indefinido, para evitar el hundimiento mayor en la pobreza de muchas familias o el paso de familias en situación de precariedad a la exclusión.

Desde luego, esta dicotomía es demasiado simple, pues olvida que la sociedad no puede dividirse en aptos y no aptos para el trabajo, conforme los baremos establecidos, de manera que existen algunas "minusvalías sociales", no recogidas por la seguridad social y que, en consecuencia, quedan en la más absoluta desprotección. Como se ha señalado, estos colectivos acuden a los servicios sociales y a su propia red social ante la expulsión del sistema. La administración pública los atiende de manera raquítica y limitada y en muchas ocasioneses incluso consciente de que aplica medidas coyunturales, que no van a transformar la sociedad y que no van a 
contribuir decisivamente a la emancipación de los individuos y las familias, sino que simplemente mantienen la paz social.

En segundo lugar, es crucial solucionar el problema de la financiación de los servicios sociales en todo el Estado, de manera que se establezca un presupuesto fijo, indefinido y actualizable. Al no ser verdaderos derechos de ciudadanía, las partidas presupuestarias asignadas para la implementación de los servicios parecen haber sido relegadas a un segundo plano en importancia. Muchos de los programas de los servicios sociales comunitarios están financiados gracias a convenios de colaboración, anuales en la mayoría de los casos, que determinan las obligaciones asumidas por cada parte para la financiación de las actividades y los medios para la puesta en marcha de los servicios y prestaciones. El compromiso para mantener tales servicios, prestaciones y el personal que los desarrolla dura el tiempo por el que se ha firmado el convenio. No existe, en consecuencia, un compromiso legal a mantener en el tiempo las actividades, servicios y prestaciones que contribuyen a la mejora de vida de la ciudadanía, especialmente en los casos más graves de pobreza y exclusión social.

La falta de continuidad en el tiempo y, especialmente, la incertidumbre sobre su misma existencia afecta muy negativamente el desarrollo del sistema. En general, la entrada al sistema público de servicios sociales de las organizaciones no lucrativas se hace, precisamente, a través de la llamada a convocatorias públicas para el desarrollo de proyectos y programas con fines sociales. Pero es muy complicado avanzar en la especialización y en la eficiencia si no está asegurada la continuidad financiera de las actividades. Aunque haya un buen diseño del sistema si no se le dota de medios suficientes es muy difícil consolidar los derechos sociales individuales y, en consecuencia, sería prolongar la atención benéfica asistencial que se ha venido prestando en España. El Estado de Bienestar se quedaría cojo pues, frente a los pilares que consagran derechos universales, el sistema de servicios sociales mantiene una dimensión focalizada y residual.

Por último, esta dinámica de funcionamiento puede absorber a los propios profesionales de los servicios sociales, convirtiéndolos en cómplices de esta situación. El exceso de trabajo y la falta de medios, además, encorsetan las posibilidades de intervención. Sin embargo, como se ha indicado anteriormente, no se debe olvidar la dimensión terapéutica del Trabajo Social, el aprovechamiento de los recursos del propio medio comunitario, así como el papel dinamizador y articulador de las redes sociales del territorio. No debería condicionarse exclusivamente la eficacia de la intervención a la existencia de recursos económicos. El Trabajo Social tiene experiencia en contextos de escasez y extrema pobreza. La mejora de la formación continuada de los profesionales y la difusión de la investigación sobre la interven- 
ción profesional es otro de los retos que hay que sumar y que contribuirán a la consolidación del sistema. En estos momentos de cambio y de aparición de nuevos riesgos sociales, es muy importante la capacidad de análisis, diagnóstico e identificación de las áreas de cambio que deben traducirse en oportunidades de mejora del funcionamiento de los servicios sociales.

Recebido em 26/8/2011 - Aprovado em 5/9/2011

\section{Bibliografía}

ADELANTADO, J.; GOMÁ, R. La política de vivienda. En: ADELANTADO, J. Cambios en el Estado del Bienestar. Políticas sociales y desigualdades en España. Barcelona: Icaria-UAB, 2000. p. 313-346.

; MORENO, R.; RECIO, C. Informe sobre la política autonómica de servicios sociales en España y Catalunya. Barcelona: Instituto de Gobierno y Políticas Públicas (IGOP) de la UBA, 2007. (Monográfico 6.)

ARRIBA, A.; PARRILLA, J.; PEREZ, B. 2009 I Congreso Estatal REPS. Recuperado el 20 jul. 2011, de Transformación de las políticas autonómicas de inclusión social: <http:// www.espanet-spain.net/congreso2009/archivos/ponencias/TP03P01.pdf>. nov. 2009.

DEFENSOR DEL PUEBLO ANDALUZ. La situación de los servicios sociales comunitarios en Andalucía. Informe especial al Parlamento. Sevilla: Defensor del Pueblo Andaluz, 2005.

FANTOVA, F. ¿Qué servicios sociales necesitamos? Ponencia inaugural de las VI Jornadas sobre Servicios Sociales Comunitarios, 2008. Logroño: Acceso 29 jul. 2011, de <www. fantova.net>.

KAHN, A. J.; KANERMAN, S. B. Los servicios sociales desde una perspectiva internacional. Madrid: Siglo XXI, 1987. (Col. Trabajo Social.)

MOLINERO, C. La política social del régimen franquista. Una asignatura pendiente de la historiografía. Ayer, n. 50, p. 319-331, 2003.

MORATA, B.; GIJON, M. T.; MATOS, R. de. O estudo da gestão pública no sistema de proteção social na Espanha. Libertas, v. 10, n. 2, p. 23-50, 2010.

; PEREZ, M.; ALARCON, J. Los servicios sociales como eje principal de las politicas autonómicas de integración, 2009. Acceso el 29 jul. 2011, en: <http://www.espanet-spain.net/congreso2009/archivos/ponencias/TP03PC01.pdf>. 
MORENO, L. La “vía media” española del modelo de bienestar mediterráneo. Papers, 63/64, 2001. Acceso el 1 jun. 2010, en: <http://ddd.uab.cat/pub/papers/02102862n63-64p67.pdf>. PELEGRÍ, X. El modelo de servicios sociales en España. Revista Internacional de Ciencias Sociales y Humanidades XVII, n. 2, p. 125-150, 2007.

RODRIGUEZ CABRERO, G. El Estado de bienestar en España: debates, desarrollos y retos. Madrid: Fundamentos, 2004.

SANZ CITORA, A. Acción social y trabajo social en España. Una revisión histórica. Acciones e investigaciones sociales, n. 13, p. 5-42, 2001.

SOLOZÁBAL, J. Sobre el Estado Autonómico Español. Revista de Estudios Políticos, n. 21, p. 105-129, 1992.

SUBIRATS, J. Los servicios sociales de atención primaria ante el cambio social. Madrid: Ministerio de Trabajo y Asuntos Sociales, 2007. 